\title{
Association of Dietary and Serum Cholesterol with Active Pulmonary Tuberculosis: A Hospital Based Study
}

\author{
J. John Prabakaran", Biniam Efrem, Matiwos Araya, Mussie Mebrahtu, \\ Henok Zeraburuk, Michael Yemane, Adiam Semere and Awet Ghebreyesus
}

\author{
Department of Biomedical Sciences (Microbiology Unit), Asmara College of Health Sciences, \\ Asmara, Eritrea, North East Africa \\ *Corresponding author
}

\begin{tabular}{|c|c|}
\hline & A B S T R A C T \\
\hline & \multirow{6}{*}{$\begin{array}{l}\text { This study was aimed at investigating the association of dietary and serum cholesterol } \\
\text { levels with active tuberculosis. A hospital based matched case control study was } \\
\text { conducted. A total of } 124 \text { participants }(62 \text { pulmonary tuberculosis (PTB) cases and } 62 \\
\text { controls) were included in the study. Out of } 62 \text { PTB cases } 28 \text { were newly diagnosed while } \\
\text { the rest } 34 \text { cases were under anti-tubercular treatment (ATT). Serum lipid and albumin } \\
\text { levels were measured from blood samples. Dietary intake of cholesterol before disease was } \\
\text { assessed by a validated food frequency questionnaire (FFQ). Smears prepared from } \\
\text { sputum samples of the newly diagnosed cases were graded for acid-fast bacilli after Ziehl- } \\
\text { Neelsen staining. Levels of serum total cholesterol (TC), low density lipoprotein } \\
\text { cholesterol (LDL-C), high density lipoprotein cholesterol (HDL-C), triglycerides (TG) and } \\
\text { albumin were significantly lower in the newly diagnosed cases when compared with the } \\
\text { controls (p<0.000) and the cases under treatment (p }<0.000) \text {. TC, HDL-C and dietary } \\
\text { cholesterol intakes differed significantly between categories of smear grades with p-value } \\
\text { of } 0.019 \text {, 0.011 and } 0.006 \text { respectively. Levels of Serum TC, HDL-C and LDL-C } \\
\text { correlated negatively with the smear grading with spear man rho of }-0.514 \text {, }-0.398 \text { and } \\
0.424 \text { respectively at p }<0.05 \text {. Reported habitual intake of cholesterol before disease also } \\
\text { correlated positively with the smear grading ( } \mathrm{r}=0.561 \text { for new cases at p }<0.05 \text { ). There was } \\
\text { a significant positive trend in the risk of developing active disease between quartiles of } \\
\text { cholesterol intake with } \mathrm{P}_{\text {trend }}=0.008 \text {. Low serum cholesterol in the new cases and its } \\
\text { negative correlation with smear grading may be due to high oxidative stress and elevated } \\
\text { cytokines reported in previous studies but levels of dietary cholesterol intake before } \\
\text { disease may have a role in fueling the development of active disease and should be } \\
\text { thoroughly studied. }\end{array}$} \\
\hline & \\
\hline $\begin{array}{l}\text { Pulmonary } \\
\text { tuberculosis, Serum } \\
\text { cholesterol, Dietary } \\
\text { cholesterol, Smear } \\
\text { grading. }\end{array}$ & \\
\hline Article Info & \\
\hline & \\
\hline & \\
\hline
\end{tabular}

\section{Introduction}

More than 2 billion people, equal to one-third of the world's population, are infected with Mycobacterium tuberculosis. Out of all the infected cases only 5-10 \% develops symptoms and in the rest, the bacterium remains latent. TB accounts for over 2 million deaths per year worldwide, making it one of the leading infectious causes of death in the world (WHO TB Control Geneva 2010; Dye et al., 2010). Cholesterol is a vital molecule for maintaining the integrity of lipid rafts making it important for the appropriate 
functioning of the immune system (Simons and Toomre, 2000). Low serum cholesterol levels were found to be associated with mortality and incidence of infectious diseases (Iribarren et al., 1998). Hypocholesterolemia is frequently found in patients with active PTB (Pérez et al., 2002). Furthermore the lowering of cholesterol in active PTB correlates negatively with severity of the disease (Deniz et al., 2007). Supplementation of cholesterol rich diet together with anttubercular treatment has been shown to accelerate bacteriologic sterilization of the sputum (Pérez et al., 2005). This finding has led authors like Pérez-Guzmán to put forward the hypothesis that hypocholesterolemia may be a major risk factor for developing active PTB (Pérez-Guzmán et al., 2006).

Contrary to such speculations cholesterol is now known to be critical in the intracellular survival of Mtb (Pandey et al., 2008). Cholesterol depletion was also shown to have an overcoming effect on the phagosome maturation block in $M$. avium infected macrophages (Chastellier et al., 2006).

Foamy macrophages (FM) are lipid loaded macrophages generated from the massive uptake of LDL and its intra-cytoplasmic accumulation as cholesterol-esters. They are classically found in granulomas (Peyron et al., 2008).

Their spatial and temporal relationship with the necrotic center has suggested a causal association between the accumulation of FM and the progress of latent infection into caseation (Russell et al., 2009). The lipids constituting the caseum in humans is rich in cholesterol, cholesterol esters and triglycerides reflecting that they are sourced from LDL which had been sequestered as lipid bodies in the foamy macrophage (Kim et al., 2010). The tissue changes that accompany granuloma progression (i.e. caseation) were also shown to be tightly associated with the up regulation of genes involved in lipid metabolism (Kim et al., 2010).

Mice fed on a high cholesterol diet were found to have a significantly higher bacterial burden in their lungs than mice on low cholesterol diet (Schäfer et al., 2009). Moreover, hypercholesterolemia due to a high cholesterol diet in mice deficient in $\mathrm{apoE}^{-/-}$ has been reported to impair the immunity to $M t b . \mathrm{ApoE}^{-/-}$mice on high cholesterol diet showed a pronounced bacterial burden, severer inflammation and died early after being aerosol infected with

Mtbwhen compared with the wild type on high cholesterol diet and $\mathrm{apoE}^{-/-}$mice on low cholesterol diet (Martens et al., 2008). Amazingly, administration of statin drug like simvastatin lowered the treatment duration in mice when taken with ATT (Dutta et.al 2016). Dietary cholesterol has also been shown to increase the risk of developing active tuberculosis in humans (Avril et al., 2016).

The role of cholesterol in the development and control of the disease, and more basically nature of the association between cholesterol and active PTB in humans are not quite clear. Do dietary and serum cholesterol levels have association with active PTB in humans? If so what kind of association do they have? The present study attempted to answer these questions first by comparing serum cholesterol levels of newly diagnosed PTB patients and those under ATT (anti-tubercular treatment) with their matched controls. The association of levels of dietary cholesterol intake and being diagnosed with active PTB were then investigated, and lastly the study investigated if there were correlations between the bacterial burden of sputum samples of participants with active PTB with their levels of serum cholesterol and amounts of habitual dietary intakes of cholesterol. 


\section{Materials and Methods}

\section{Study population and design}

A total of 124 participants who visited Halibet and Orrota referral hospitals in Asmara the capital city of Eritrea, between January $10^{\text {th }}$ and May $30^{\text {th }} 2016$ were examined. Out of 124 participants 62 patients reporting themselves with clinical manifestations suggestive of pulmonary TB together with the finding of abnormal chest X-ray, and the demonstration of acid fast bacilli by Ziehl-Neelsen method of staining on their sputum smears were included as cases. Patients diagnosed with PTB but having diabetes mellitus and HIV infection were excluded from the study. Those who were smear negative but whose final diagnosis was pulmonary TB were also included as cases. Out of 62 cases 34 cases that had been previously diagnosed with active TB and were under anti-tuberculosis treatment (ATT) at the health centers with no previous history of diabetes mellitus and HIV were also included as cases. The PTB positive cases were then sub divided into two groups (i.e. the newly diagnosed and those under ATT) to see the possible effect of treatment and recovery on serum lipid levels. Sixty four healthy people who visited the same settings for conditions other than TB were selected as control based on their matching statues for age, sex and body mass index (BMI) with the cases. Having diabetes mellitus, HIV infection, liver diseases, respiratory disease or symptoms related with $\mathrm{TB}$, lactating or pregnant women, use of cholesterol lowering medications, history of being diseased with tuberculosis and history of tuberculosis infection in one or more of their direct family members were used as exclusion criteria's for the controls. This study has got its ethical clearance from the Ministry of Health $(\mathrm{MoH})$ research ethical committee. Informed consent was obtained from each individual included in the study. Serum cholesterol levelsand habitual intakes of cholesterol along with frequency of consumption of other foods were assessed for all the participants. Extent of bacterial burden in the sputum of the newly diagnosed PTB positive cases was assessed by smear grading while the smear grade at diagnosis for those under ATT was obtained from the patient card. Socio-demographic characteristics, BMI, alcohol consumption and smoking statues were also assessed for all participants.

\section{Bacteriological examination}

Bacteriologic examination was carried out on three sputum samples collected on two consecutive days for the new cases. The sputum smears were examined for acid-fast bacilli using Ziehl-Neelsen method of staining (WHO Geneva, 1998). The smear was prepared from the purulent and mucoid part of the sample. The bacterial burden of the sputum samples was determined by degree of smear positivity after examining 100 high power fields (HPF). The finding of 1-9 AFB/100 HPF and smear negative cases were graded as "-/ (1-9)", 10-99 AFB/100 HPF as "+", 1-10 AFB/HPF as "++" and $>10$ $\mathrm{AFB} / \mathrm{HPF}$ as "+++". The grading was confirmed by an experienced lab-technician. Discrepant results were resolved by a third lab-technician. For those under treatment, their smear results at diagnosis were obtained from the patient card.

\section{Measurement of biochemical parameters}

Peripheral venous blood was drawn from fasting participants by vein puncture from the anticubital area. Six milliliter of blood specimen was then collected in blank sterile vacutainers. Serum sample was separated by centrifugation after ensuring the blood has clotted. Sample was then delivered to the National Health Laboratory (NHL). Serum cholesterol levels were then determined by a 
daily calibrated and quality controlled automated Beckman-AU480clinical chemistry analyzer. Serum levels of total cholesterol (TC), high density lipoprotein cholesterol (HDL-C), triglycerides (TG) and albumin were measured. Low density lipoprotein cholesterol (LDL-C) was determined using the Friedewald formula.

\section{Dietary assessment}

Information on dietary intakes was gathered by an interviewer administered 42 item semiquantitative FFQ (food frequency questionnaire) specifically developed for this study. It was used to assess the participant's usual diet over the past 12 months before diagnosis for cases and 12 months before the interview for the controls. Since there were no previously established FFQ for our study population the FFQ was developed and validated before the initiation of this study. It was developed in two stages; the first stage involved the selection of commonly consumed foods by interviewing some of the people visiting the above mentioned study sites. After the selection of commonly consumed foods the prepared FFQ was validated for its ability to estimate cholesterol intake with the use of a two day 24-hour dietary recall interview on 30 people. The reference time of the questionnaire was reduced to one month to enable comparison with a two day 24-hour diet recall extended over a month. The days selected for the 24hour recall were Sunday and Thursday. Its reliability was assessed by test and retest method. Cholesterol intake estimated by $24-$ hour diet recall and our FFQ showed a Pearson's correlation coefficient of $r=0.569$ at $p<0.05$ and $r=0.723$ at $p<0.05$ between the repeatedly administrated FFQ, which is considered good for this purpose.

For each food item in the FFQ participants were asked to select their average frequency of intake from 11 possible responses, ranging from never up to two or more times per day. A commonly used unit or portion size was specified, and participants were asked how many of that specified unit or portion they habitually consume during a single meal. Some published reports (Carlos and Jorge 2010; Scherr et al., 2009) as well as the Matvaretabellens food composition database was used to estimate the cholesterol content of each cholesterol containing food component (i.e. a component approach was used). Average daily intake of cholesterol was computed by multiplying the consumption frequency of each food by the cholesterol content of the specified portion size. Total average cholesterol intake was then taken as the sum of the results.

The consumption frequency of different food groups (cereals, vegetables, legumes, dairy products, egg, fish, meat, fruits, tea and coffee) were also converted into frequency of consumption per week for them to be included in further analysis.

\section{Statistical analysis}

The collected data was analyzed by SPSS software version 20. Normally distributed parameters were tested by paired t-test while Wilcoxon signed rank sum test and MannWhitney U test were done for non-normally distributed measures. Spearman's correlation and partial correlation coefficients were used to evaluate the association of dietary cholesterol intake and serum lipid levels with the degree of smear positivity for AFB.ANOVA and Kruskal-Wallis test were used to compare categories of smear grades. McNemar's test was used to evaluate associations between categorical variables of the matched cases and controls. The study participants were categorized into quartiles of cholesterol intake which were was represented by their median value. 
Conditional logistic regression was then used to estimate the odds ratio adjusted for possible confounders for each quartile in reference to the lowest quartile. The median values of each quartile were entered as continuous variable to analyze the existence of a trend in the odds ratio. Variables included in the model were selected by the purposeful variable selection method as proposed by Hosmer and Lemeshow (1999, 2000) as it proved to be more sensitive for including confounders. The last model was checked for multicolliniarity by running it in linear regression to check for the VIF, tolerance and eigenvalue of the predictors.

\section{Results and Discussion}

The study included a total of 124 participants which consisted of 62 tuberculosis cases and 62 age ( \pm 2 years), sex and BMI matched controls. From the 62 participants included as cases 28 were newly diagnosed pulmonary TB cases while the rest 34 cases were under ATT. Table 1 shows the socio-demographic characteristics of the study participants. The cases and controls were comparable on the matching variables of age and sex. The matching for BMI was not strictly adhered in some of the newly diagnosed cases as some were severely malnourished and finding a suitable control proved to be difficult. Although there was non-significant difference in the number of people who were under nourished (i.e. $B M I<18.5 \mathrm{~kg} / \mathrm{m}), \quad p=0.829$. Further analysis by grading the level of malnutrition indicated by BMI showed a higher number of severely malnourished cases than controls (Table 2). Wilcoxon signed rank sum test showed that BMI was significantly lower in the cases than their controls at $p=$ 0.026 (not shown). There was no significant difference in the ethnicity, religious affiliation, residence and current family members of the cases and controls. Unemployment was significantly higher in the cases than the controls at $p=0.017$. Despite the insignificant difference in the distribution of cases and controls with respect to educational level the isolated comparison of the number of cases with higher education levels (i.e. educational level above $12^{\text {th }}$ grade) was significantly higher in the controls than the cases with a $p=0.031$ (not shown). The prevalence of reported smoking habit was low in our study sample only $(1.6 \%)$ of the cases and $(4.9 \%)$ of the controls reported positively.

The comparison of serum lipid and albumin levels was done separately for the newly diagnosed and those under treatment with their age, sex and BMI matched controls. The evaluation of the data normality and homogeneity of serum lipids and albumin levels in the newly diagnosed cases and their controls showed that the levels of TC and LDL were normally distributed as indicated by normal Q-Q plots and insignificant deviation from normality by KolmogorovSmirnov and Shapiro-Wilk tests of normality. The levels of skewness and Kurtosis were acceptable at $p>0.05$. The slightly skewed distribution of TG was normalized upon logarithmic transformation. Levels of HDL and Albumin were not normally distributed even after transformations. The test for homogeneity by Levine's test showed no significant difference in the variance between the cases and controls for all serum lipid levels. But it was significant for albumin levels. The comparison of serum levels of TC, LDL-C and TG was therefore done by paired t-test while Wilcoxon signed rank sum test was used for the others. The comparison showed significantly lower levels of all serum lipids and Albumin levels when compared with the matched controls at $p<0.000$ (Table 3 ). The evaluation of the normality for the biochemical parameters prior to comparing cases under ATT and their controls showed a normal distribution for Albumin and HDL-C levels. Levels of TC and LDL-C were 
normalized upon inverse transformation while levels of TG were normalized upon logarithmic transformation. The Levine's test for homogeneity showed no significant difference in the variance for all the parameters. Paired-t test was therefore used to compare serum lipid and albumin levels between the cases under treatment and their matched controls. The analysis showed no significant difference in the levels of TC, HDL-C and LDL-C ( $>0.05)$. The difference was significant only for levels of triglycerides and albumin, which was significantly lower in the treatment cases as shown in the table 3 at $\mathrm{p}<0.05$ for both parameters.

Lipid and albumin levels between the newly diagnosed (i.e. those that had not started ATT) with those that had started treatment were also compared. Serum levels of TC, HDL-C, LDL-C, TG and Albumin showed a normal distribution when logarithmically transformed satisfying the normality assumption. Independent $t$-test was done and the results showed that serum lipids and albumin, with the exception of triglycerides were significantly higher in the cases under treatment $(\mathrm{p}<0.000)$ as shown in table 3.

Grouping of the cases based upon their duration on treatment showed a significant linear increase with $\mathrm{P}_{\text {trend }}<0.000$ and a nonsignificant deviation from linearity in the mean levels of HDL-C, TC, LDL-C and albumin levels (Figure 1).

Although age, sex and BMI were matched a model was constructed to control for other possible confounders. Conditional logistic regression was used to model the data. Purposeful variable selection method was used to select the variables. Univariate analysis was done at $p$-value cut-off point of 0.25 , as more traditional levels like 0.05 can fail in identifying variables known to be important (Bendel et al., 1999; Mickey et al.,
1989). Higher educational level, employment statues, legume, vegetable, and tea consumption, quartiles of cholesterol intake, residence, meat and fruit consumption were selected as candidates for the multivariate analysis in the model with $\mathrm{p}<0.25$.

In the iterative process of variable selection, covariates were removed from the model if they were non-significant at the 0.1 alpha level and non-confounder evaluated as a change in any remaining parameter estimate greater than $15 \%$ as compared to the full model. At the end of this iterative process quartiles of cholesterol intake, legume consumption (frequency of consumption per week), vegetable consumption (frequency of consumption per week), employment statues (employed and non-employed), Higher educational level (Educational level above $12^{\text {th }}$ grade or below) and meat consumption (frequency of meat consumption per week) were kept in the final model (Table 4). None of the previously excluded variables made it to the final model after they were added back one at a time. For more details on the purposeful selection process used in this study, refer to Hosmer and Leme show (1999). The diagnosis of the model for multi colliniarity didn't show any problems as all VIF values were below 1.5 and minimum tolerance value of 0.709 . None of the eigenvalue was zero.

The test for a trend in the odds ratio between with increasing cholesterol intake was done by entering the median values of each quartiles of intake as a pseudo continuous variable into the model. Running the model showed a significant linear trend in the odds ratio with the increase in the quartile of cholesterol intake with $\mathrm{P}_{\text {trend }}$ 0.008. The modeling showed a positive association of the odds ratio with the increase of cholesterol in the diet. The results of our analysis indicate a dose response association of the risk of 
developing active tuberculosis with increase in the cholesterol intake. To assess the possibility of some recall bias introduced to the model due to including the cases under treatment the model was run by excluding the cases under treatment from the model. Only 28 pairs remained for analysis which was small to accommodate all the previously used variables. We therefore used unconditioned logistic regression to include the controls for the cases under treatment. The association was not changed with the exclusion of the cases under treatment with a $\mathrm{P}_{\text {trend }}=0.004$.

Estimated cholesterol intake obtained from the FFQ was used to assess the association of dietary cholesterol with the risk of developing active PTB. The unadjusted chi square test showed no significant association of the categories of cholesterol intake with TB at $\mathrm{p}>0.05$. There was a slight increase in the crude odds ratio with increasing quartiles of intake using the lowest quartile as the reference exposure. Calculation of the adjusted odds ratio using the final model showed an increase in the odds ratio with the increase in the quartiles of cholesterol intake. Being in the highest quartile of cholesterol intake was associated with an8.57 (95\% CI 1.586-46.35) times risk of having the active form of the disease when compared with the first quartile of intake (Table 5).

Table.1 Socio-demographic characteristics of study participants

\begin{tabular}{|c|c|c|c|c|}
\hline \multicolumn{2}{|c|}{ Socio-demographic variable } & \multirow{2}{*}{$\frac{\text { Cases }}{46.8 \%}$} & \multirow{2}{*}{$\begin{array}{l}\text { Controls } \\
47.5 \%\end{array}$} & \multirow{2}{*}{$\begin{array}{l}\text { p-value } \\
0.975\end{array}$} \\
\hline \multirow[t]{3}{*}{ Age (years) } & $17-37$ & & & \\
\hline & $38-58$ & $38.7 \%$ & $39.3 \%$ & \\
\hline & $59-86$ & $14.5 \%$ & $13.1 \%$ & \\
\hline \multirow[t]{2}{*}{ Sex } & Male & $38.7 \%$ & $37.7 \%$ & \multirow[t]{2}{*}{0.998} \\
\hline & Female & $61.3 \%$ & $62.3 \%$ & \\
\hline \multirow[t]{2}{*}{ BMI } & $<18.5$ & $59.3 \%$ & $57.4 \%$ & \multirow[t]{2}{*}{0.829} \\
\hline & $>18.5$ & $40.7 \%$ & $42.6 \%$ & \\
\hline \multirow[t]{4}{*}{ Educational level } & None & $14.5 \%$ & $6.6 \%$ & \multirow[t]{4}{*}{0.062} \\
\hline & Primary $(1-8)$ & $45.2 \%$ & $39.3 \%$ & \\
\hline & Secondary $(9-12)$ & $32.3 \%$ & $29.5 \%$ & \\
\hline & Higher $(>12)$ & $8.1 \%$ & $24.6 \%$ & \\
\hline \multirow[t]{4}{*}{ Ethnicity } & Tigrina & $92.9 \%$ & $91.8 \%$ & \multirow[t]{4}{*}{0.554} \\
\hline & Tigre & $7.1 \%$ & $4.9 \%$ & \\
\hline & Saho & $0 \%$ & $1.6 \%$ & \\
\hline & Others & $0 \%$ & $1.6 \%$ & \\
\hline \multirow[t]{4}{*}{ Religion } & Orthodox & $88.5 \%$ & $80 \%$ & \multirow[t]{4}{*}{0.608} \\
\hline & Muslim & $7 \%$ & $15 \%$ & \\
\hline & Catholic & $1.9 \%$ & $3.3 \%$ & \\
\hline & Others & $1.9 \%$ & $1.7 \%$ & \\
\hline \multirow[t]{2}{*}{ Residence } & Urban & $78 \%$ & $84.7 \%$ & \multirow[t]{2}{*}{0.344} \\
\hline & Rural & $22 \%$ & $15.3 \%$ & \\
\hline \multirow[t]{2}{*}{ Employment } & Unemployed & $50 \%$ & $29 \%$ & \multirow[t]{2}{*}{$0.017^{*}$} \\
\hline & Employed & $50 \%$ & $71 \%$ & \\
\hline \multirow[t]{2}{*}{ Smoking habit } & Yes & $1.6 \%$ & $4.8 \%$ & \multirow[t]{2}{*}{0.309} \\
\hline & No & $98.4 \%$ & $95.2 \%$ & \\
\hline \multirow[t]{2}{*}{ Current family members } & $<6$ & $72.6 \%$ & $63.6 \%$ & \multirow[t]{2}{*}{0.299} \\
\hline & $>6$ & $27.4 \%$ & $36.4 \%$ & \\
\hline
\end{tabular}

*Significant at $\mathrm{p}<0.05$ 
Table.2 Comparison of cases and controls on the degree of malnutrition

\begin{tabular}{|l|l|l|l|l|l|}
\hline BMI (Kg/M) & $\begin{array}{l}\text { Degree of } \\
\text { Malnutrition }\end{array}$ & New cases & $\begin{array}{l}\text { Matched } \\
\text { controls }\end{array}$ & $\begin{array}{l}\text { Treatment } \\
\text { cases }\end{array}$ & $\begin{array}{l}\text { Matched } \\
\text { controls }\end{array}$ \\
\hline$<16$ & Severe malnutrition & $28.6 \%$ & $14.3 \%$ & $23.5 \%$ & $8.8 \%$ \\
\hline $16-17$ & Moderate malnutrition & $14.3 \%$ & $10.7 \%$ & $8.8 \%$ & $8.8 \%$ \\
\hline$>17-18.5$ & Mild malnutrition & $32.1 \%$ & $50 \%$ & $14.7 \%$ & $26.5 \%$ \\
\hline$>18.5-25$ & Normal & $25 \%$ & $25 \%$ & $52.9 \%$ & $55.9 \%$ \\
\hline
\end{tabular}

Table.3 Comparison of serum levels of TC, HDL-C, LDL-C, TG and Albumin

\begin{tabular}{|l|l|l|l|}
\hline Variables & Newly diagnosed $(\boldsymbol{M} \pm \boldsymbol{S D})$ & Matched controls $(\boldsymbol{M} \pm \boldsymbol{S D})$ & p-value \\
\hline TC & $98.96 \pm 32.96(\mathrm{mg} / \mathrm{dl})$ & $153.35 \pm 32.8(\mathrm{mg} / \mathrm{dl})$ & $\mathbf{0 . 0 0 0}$ \\
\hline HDL-C & $22.21 \pm 14.57(\mathrm{mg} / \mathrm{dl})$ & $44.98 \pm 13.95(\mathrm{mg} / \mathrm{dl})$ & $\mathbf{0 . 0 0 0}$ \\
\hline LDL-C & $61.78 \pm 23.48(\mathrm{mg} / \mathrm{dl})$ & $88.98 \pm 22.17(\mathrm{mg} / \mathrm{dl})$ & $\mathbf{0 . 0 0 0}$ \\
\hline TG & $74.67 \pm 27.8(\mathrm{mg} / \mathrm{dl})$ & $96.96 \pm 38.34(\mathrm{mg} / \mathrm{dl})$ & $\mathbf{0 . 0 1 8}$ \\
\hline Alb & $2.71 \pm 0.74(\mathrm{~g} / \mathrm{dl})$ & $4.09 \pm 0.468(\mathrm{~g} / \mathrm{dl})$ & $\mathbf{0 . 0 0 0}$ \\
\hline & Treatment cases $(\boldsymbol{M} \pm$ SD $)$ & Matched controls $(\boldsymbol{M} \pm$ SD $)$ & \\
\hline TC & $171.41 \pm 47.02$ & $154.06 \pm 37.88$ & $\mathbf{0 . 4 0 1}$ \\
\hline HDL-C & $43.15 \pm 12.55$ & $38.33 \pm 9.82$ & $\mathbf{0 . 3 2 6}$ \\
\hline LDL-C & $109.75 \pm 43.91$ & $97.31 \pm 34.42$ & $\mathbf{0 . 6 5 6}$ \\
\hline TG & $87.38 \pm 34.16$ & $106.7 \pm 51.04$ & $\mathbf{0 . 0 0 6}$ \\
\hline Alb & $3.51 \pm 0.63$ & $3.86 \pm 0.42$ & $\mathbf{0 . 0 4 4}$ \\
\hline & Newly diagnosed cases & Cases under treatment & \\
\hline TC & $98.96 \pm 32.96$ & $171.41 \pm 77.02$ & $\mathbf{0 . 0 0 0}$ \\
\hline HDL-C & $22.21 \pm 14.57$ & $43.15 \pm 12.55$ & $\mathbf{0 . 0 0 0}$ \\
\hline LDL-C & $61.78 \pm 23.48$ & $109.75 \pm 43.91$ & $\mathbf{0 . 0 0 0}$ \\
\hline TG & $74.67 \pm 27.8$ & $87.38 \pm 34.16$ & $\mathbf{0 . 3 9 0}$ \\
\hline Alb & $2.71 \pm 0.74$ & $3.51 \pm 0.63$ & $\mathbf{0 . 0 0 0}$ \\
\hline
\end{tabular}

$\mathrm{M} \pm \mathrm{SD}$ - Mean \pm Standard Deviation

Table.4 Final model of the conditional logistic regression used for testing for a trend by adjusting for confounders

\begin{tabular}{|c|c|c|c|c|c|c|c|}
\hline $\begin{array}{l}\text { Variables in the final } \\
\text { Model }\end{array}$ & B & SE & Wald & Sig. & $\begin{array}{l}\operatorname{Exp} \\
(\beta)\end{array}$ & \multicolumn{2}{|c|}{$\begin{array}{l}\text { 95\% CI for } \operatorname{Exp}(\beta) \\
\text { Lower Upper }\end{array}$} \\
\hline Employment & 1.697 & 0.753 & 5.073 & $0.024 *$ & 5.456 & 1.246 & 23.886 \\
\hline Legumes & 0.154 & 0.088 & 3.098 & 0.078 & 1.167 & 0.983 & 1.385 \\
\hline Vegetables & -0.225 & 0.092 & 6.039 & $0.014 *$ & 0.798 & 0.667 & 0.995 \\
\hline Meat & -0.151 & 0.087 & 2.974 & 0.085 & 0.860 & 0.725 & 1.021 \\
\hline $\begin{array}{l}\text { Quartile of } \\
\text { cholesterol intake }\end{array}$ & 0.016 & 0.006 & 6.941 & $0.008 *$ & 1.016 & 1.004 & 1.027 \\
\hline $\begin{array}{l}\text { Higher educational } \\
\text { level }\left(>12^{\text {th }}\right)\end{array}$ & -1.042 & 0.798 & 1.703 & 0.192 & 0.353 & 0.074 & 1.687 \\
\hline
\end{tabular}

*significant at $\mathrm{p}<0.05$ 
Table.5 Comparison of the odds ratio of the higher quartiles of intake with the first quartile

\begin{tabular}{|l|l|l|l|l|}
\hline Quartile of intake & Crude OR & 95\% CI & Adjusted OR & 95\% CI \\
\hline Quartile 1 (15.56mg/day) & 1 & & 1 & \\
\hline Quartile 2 (41.79 mg/day) & 1.498 & $0.529-4.218$ & 1.11 & $0.301-4.1$ \\
\hline Quartile 3 (67.97 mg/day) & 1.316 & $0.428-4.041$ & 1.49 & $0.371-7.015$ \\
\hline Quartile 4 (159.12 mg/day) & 1.936 & $0.704-5.324$ & 8.573 & $1.586-46.351$ \\
\hline
\end{tabular}

OR-odds ratio CI-Cholesterol Intake

Table.6 ANOVA and Kruskal-Wallis analysis of mean values of serum lipids, albumin, Estimated Cholesterol Intake (ECI), BMI andAge with categories of smear grades

\begin{tabular}{|l|l|l|l|l|l|}
\hline Variables & \multicolumn{4}{|l|}{ AFB Smear Grading } & P-value \\
\hline & $\begin{array}{l}\boldsymbol{-}(\mathbf{1 - 9}) \\
\mathbf{n}=\mathbf{5}\end{array}$ & $\begin{array}{l}\mathbf{n}=\mathbf{5} \\
\mathbf{n}\end{array}$ & $\begin{array}{l}\mathbf{+ +} \\
\mathbf{n = 5}\end{array}$ & $\begin{array}{l}\mathbf{+ + +} \\
\mathbf{n = 1 3}\end{array}$ & \\
\hline TC & $133.2 \pm 26.53$ & $101.2 \pm 31.4$ & $106.2 \pm 28.14$ & $82.1 \pm 28.57$ & $\mathbf{0 . 0 1 9}$ \\
\hline LDL-C & $76.7 \pm 20.68$ & $71.09 \pm 27.9$ & $65.5 \pm 13.56$ & $51.02 \pm 22.69$ & $\mathbf{0 . 1 3 0}$ \\
\hline TG & $72.2 \pm 35.32$ & $78.2 \pm 12.45$ & $72.2 \pm 34.76$ & $75.23 \pm 29.3$ & $\mathbf{0 . 9 8 5}$ \\
\hline HDL-C & $41.99 \pm 16.21$ & $14.47 \pm 4.84$ & $26.25 \pm 12.06$ & $16.08 \pm 10.1$ & $\mathbf{0 . 0 1 1}$ \\
\hline Albumin & $3.36 \pm 0.61$ & $2.53 \pm 0.78$ & $2.92 \pm 0.507$ & $2.46 \pm 0.74$ & $\mathbf{0 . 1 1 4}$ \\
\hline ECI** & $26.8 \pm 19.5$ & $48.6 \pm 61.9$ & $117.7 \pm 62.18$ & $124.9 \pm 64.6$ & $\mathbf{0 . 0 0 6} *$ \\
\hline BMI & $17.6 \pm 1.89$ & $18.68 \pm 0.319$ & $17.1 \pm 1.89$ & $16.3 \pm 1.92$ & $\mathbf{0 . 0 6 4}$ \\
\hline Age & $39.0 \pm 15.7$ & $37.6 \pm 17.03$ & $35.0 \pm 7.0$ & $39.0 \pm 15.7$ & $\mathbf{0 . 9 9 9 *}$ \\
\hline
\end{tabular}

*Kruskal-Wallis test was used. **ECI: Estimated Cholesterol Intake before disease

Table.7 Correlation of serum lipid, albumin levels and quartiles of cholesterol intake with AFB smears grading using the Spearman correlation and partial correlation in the newly diagnosed cases

\begin{tabular}{|l|l|l|l|l|l|l|l|l|l|}
\hline $\mathbf{N = 2 8}$ & TC & HDL-C & LDL-C & TG & ALB & BMI & Age & QCI \\
\hline $\begin{array}{l}\text { Smear } \\
\text { grading }\end{array}$ & Spearman's rho & -0.513 & -0.388 & -0.428 & 0.034 & -0.363 & -0.380 & -0.012 & 0.564 \\
\cline { 2 - 10 } & $\begin{array}{l}\text { Partial correlation } \\
\text { coefficient }\end{array}$ & -0.611 & -0.639 & -0.446 & -0.075 & -0.407 & & & 0.639 \\
\hline P-value for Spearman correlation & 0.005 & 0.041 & 0.023 & 0.866 & 0.057 & 0.046 & 0.953 & 0.002 \\
\hline P-value for partial correlation & 0.001 & 0.001 & 0.025 & 0.721 & 0.044 & & & 0.001 \\
\hline
\end{tabular}

QCI -Quartiles of Cholesterol Intake ALB-Albumin

Fig.1 Comparison of mean levels of HDL, TC, LDL and TG when cases were grouped based upon their duration of treatment. All except TG levels showed a significant linear increase.

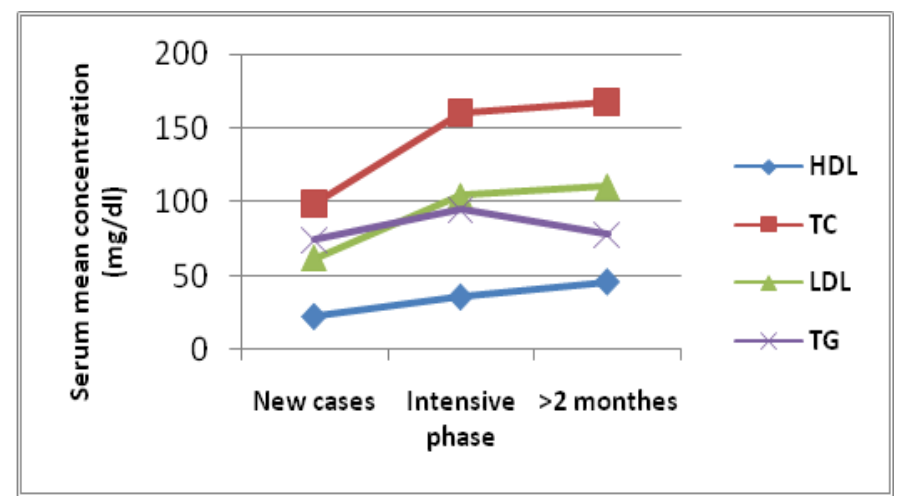


Fig.2a The correlation of serum lipid levels with the degree of smear grading
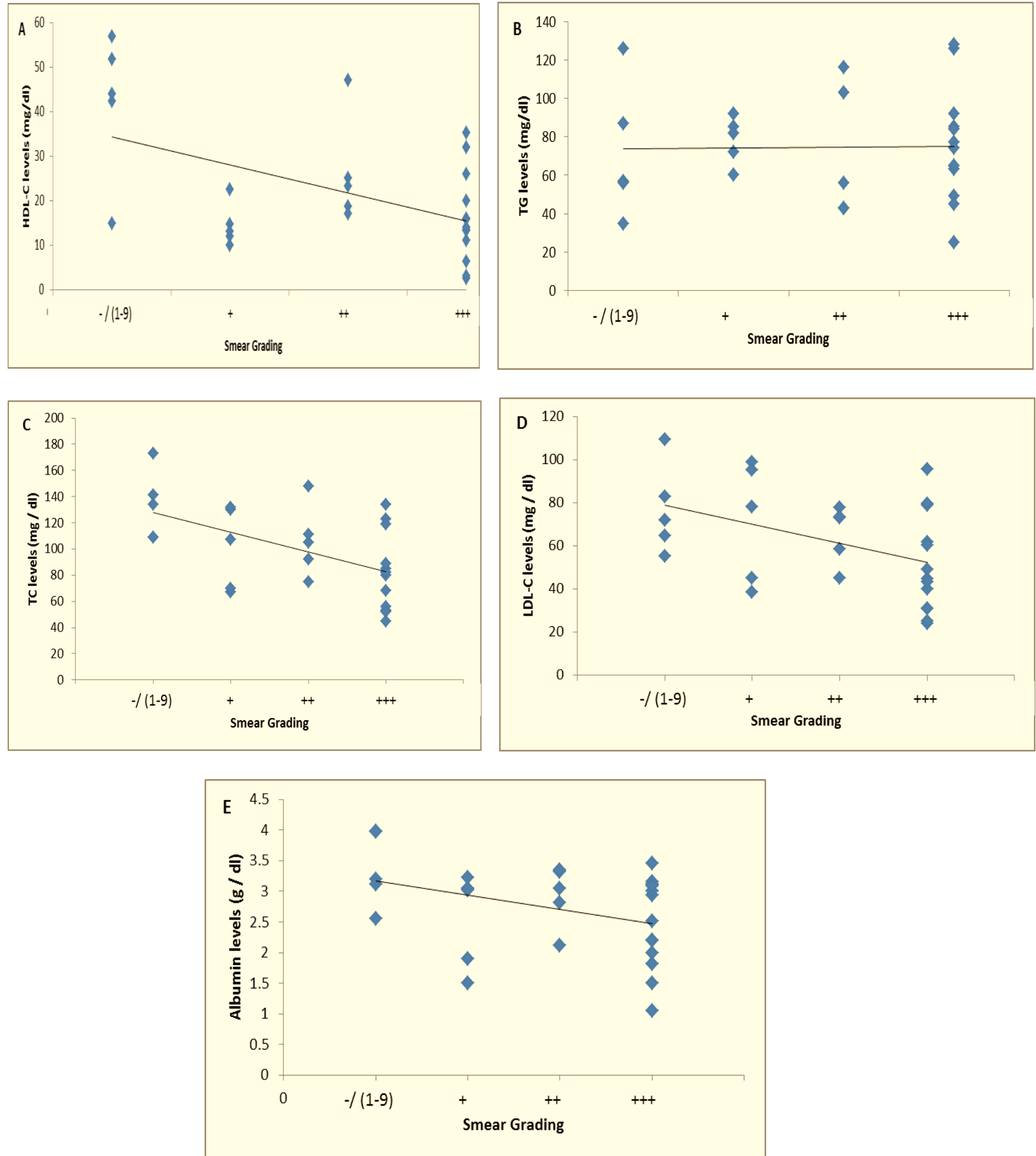

(A) A negative correlation between serum LDL-Cholesterol and AFB smear grading (B) A negative correlation between HDL-C and AFB smear grading. (C) A negative correlation between TC and AFB smear grading. (D) No correlation between TG and AFB smear grading (E) a negative correlation but non-significant correlation of albumin with AFB smear grading 
Fig.2b The correlation of estimated cholesterol intake and AFB smear grade
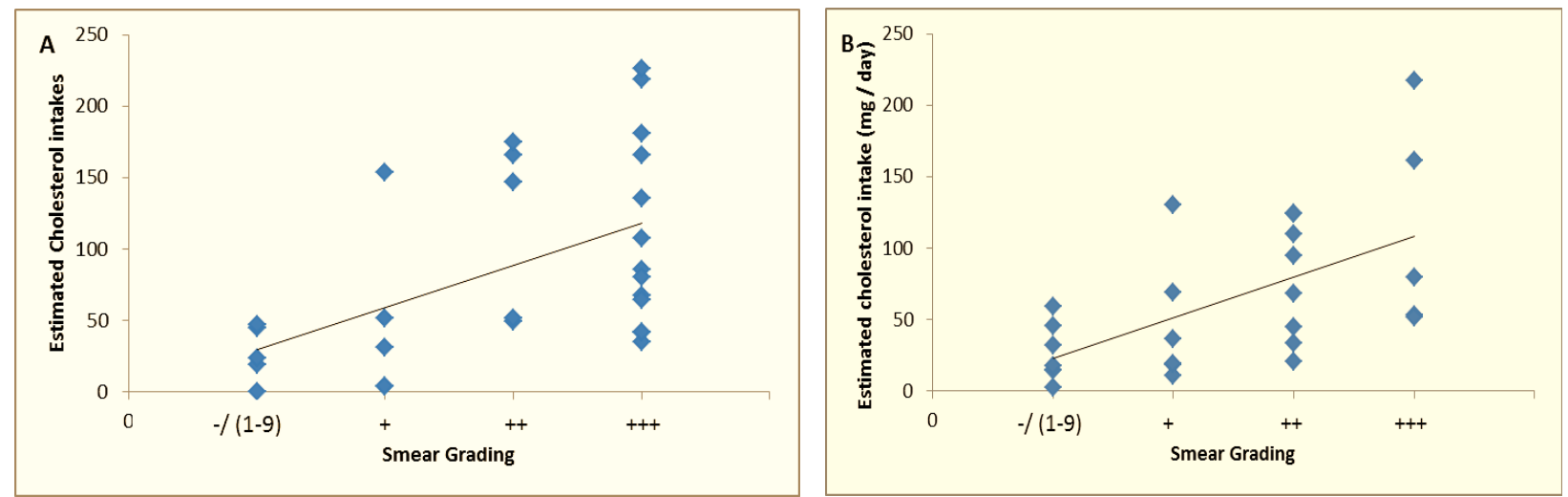

(A) Correlation of smear grading with estimated cholesterol intake showed a positive correlation in the newly diagnosed cases.

(B) Correlation of smear grading with the estimated cholesterol intake in the cases under ATT.

The extent of bacterial burden in the sputum of the cases at the time of diagnosis was analyzed for its possible associations with serum lipid levels and dietary intake of cholesterol. There was no significant difference in the homogeneity of variance for all the measured parameters. The presence of possible association was first analyzed by ANOVA for levels of TC, LDL-C and TG which had normal distribution with in the newly diagnosed cases $(n=28)$. Albumin and HDL-C levels were not distributed normally and were analyzed by Kruskal-Wallis test. There was a significant difference in serum levels of TC, HDL-C and estimated dietary cholesterol intakes across different groups of smear grading's. Other parameters like the levels of LDL-C, TG, BMI and age were not significant across the categories of smear grading (Table 6).

There was a significant linear decrease $(p<$ 0.05 ) in serum lipids of LDL-C, TC, Albumin and BMI with higher smear grading without deviating from normality $(p>0.05)$, while serum levels of HDL-C were significantly deviated from linearity. There was also a significant linear increase $(p=0.002)$ in the mean levels of cholesterol intake before disease with increase of smear grading without significant deviation from linearity ( $p$ $=0.601)$.

The strength and direction of the correlation between serum levels of the biomolecules and smear grading was analyzed by Spearman's rho and partial correlation was used to assess the correlation after controlling for age, sex and BMI. It was found that levels of TC, HDL-C and LDL-C had a significant negative correlation $(p<0.05)$ with the extent of the smear grading (Table 7) when analyzed by Spearman's rho. Partial correlation coefficient showed similar results; HDL-C, LDL-C and TC levels showed a significant negative correlation with smear grading with coefficients of $-0.639,-0.446$ and -0.611 respectively all at $p<0.05$. Such correlation was again not apparent for TG levels. Quartiles of cholesterol intake (QCI) had a significant correlation with Spearman's rho of 0.561 at $p=0.002$. Similar results were obtained upon analysis by partial correlation with a coefficient of 0.639 at $p=0.001$. Increased levels of cholesterol intake were correlated with higher degree of smear grading. Similar results were obtained for the recalled report of cholesterol intakes for the cases under ATT with their smear grade at diagnosis with Spearman rho of 0.598 at $p=$ 
0.001. The partial correlation coefficient was similarly significant with a value of 0.466 at $p$ $=0.033$ (Figure 2).

Tuberculosis still persists as a major health problem throughout the world causing a large number of annual deaths worldwide (Dye et $a l ., 2010)$. The focus of the present study was solely concerned with the association of both dietary and serum cholesterol with active pulmonary TB. Hypocholesterolemia had been proposed to be a risk factor for developing active tuberculosis (Pérez et al., 2006). This was based on the facts that hypocholesterolemia appears to co-occur with active tuberculosis in several cross-sectional and case-control studies, its degree of lowering also varies with the severity of the disease (Deniz et al., 2007). Experiment evaluating the effects of cholesterol supplementation during ATT showed that it accelerated the rate of culture conversion but did not influence the symptoms (Pérez et al., 2006). However, studies in apoE ${ }^{-/}$deficient mice showed that those fed cholesterol rich foods showed higher bacterial burdens and died early (Martens et al., 2008). Administration of statin drug like simvastatin was also reported to lower the treatment duration in mice when taken with ATT (Dutta et al., 2016). All these may be related with the fact that cholesterol is critical for intracellular survival of $M t b$ in the host cell (Pandey et al., 2008). Studies showing that cholesterol depletion of cells could overcome the pathogen induced phagosome maturation and autophagy block provided further support to the claims (Chastellier et al., 2006).In the present study it was claimed that both serum and dietary cholesterol have association with the development and outcome of active tuberculosis on humans. We were not able to predict the nature of the association as the literature provides no clear and consistent association. The results of this study showed that serum cholesterol levels besides being significantly lower in the newly diagnosed cases, correlated negatively with the extent of bacterial load of their sputum sample. Dietary cholesterol on the other hand appeared to have a significant positive correlation with both the risk of developing active disease and the extent of the bacterial load in the sputum sample.

The finding of low serum cholesterol levels in the newly diagnosed cases confirmed the finding of previous studies (Pérez Guzmán et al., 2002, Akiibinu 2008, and Kassim et al., 2012). High degree of oxidative stress had been reported to occur during active tuberculosis (Tesfaye et al., 2003, Samuel 2013). This may in part provide an explanation for the presence of low lipid levels because lipid peroxidation due to the oxidative stress is shown to cause lowering of serum lipid levels (Sasaki et al., 1994). Studies showing the presence of higher levels of serum malondialdehyde, a lipid peroxidation product in active PTB patients may provide further evidence to these phenomena (Tesfaye et al., 2003, Samuel 2013). This issue may be addressed in the present study by the presence of lower albumin (natural antioxidant) in those with active pulmonary tuberculosis. Proinflammatory cytokines like IL-6, IL-1, TNF$\alpha$ and IFN's which are known to down regulate serum lipid levels by interfering withlipid metabolism (Vanet.al 1994; Henrich 1990) have also been shown to increase during the onset of active tuberculosis (FigenDeveci et al., 2005).

The cases under ATT had comparable levels of serum lipids with their controls except that of TG and albumin which were significantly lower. The same comparison showed that cases under ATT had significantly higher levels of TC, HDL-C, LDL-C and albumin than the newly diagnosed cases except for TG which was non-significant. These results may 
imply that the lowered levels of serum lipids revert or normalize upon starting ATT. Our results agree with the previously done studies where levels of TC showed a significant increase after 8 weeks of follow up (Mohamed et al., 2012).Similarly levels of TC and HDL-C showed a significant increase with treatment after 6 months of follow up (Akpovi et al., 2013). There was no significant increase in the levels of TG before and after treatment for both studies which again agrees with our results. Unlike these studies which found no significant increase for levels of LDL-C, it was significantly higher in cases under ATT than the newly diagnosed cases in this study. The pre-post design of those studies where the cases act as their own control may give more reliable result than our approach of comparing groups of matched individuals. The duration of treatment was also not kept constant in our study which could be a source of variation. The observed linear increase in the mean levels of TC, HDL-C, and LDL-C with duration under treatment (Fig. 1) may together with the above observation oppose the claim that lowering of serum lipids may be the cause of the disease (Pérez et al., 2006) but make it more likely to be a consequence of the disease.

After adjusting for potential confounders, being in the highest quartile of cholesterol intake was associated with an8.57 (95\% CI 1.586-46.35) times risk of having the active PTB when compared with the first quartile of intake. The test for a linear trend in the odds ratio was significant at $P_{\text {trend }}=0.008$ showing a dose response association between the increase in the quartiles of cholesterol intake and the risk of developing active PTB. A recently published prospective cohort study found dietary cholesterol to be positively associated with the risk of developing active tuberculosis (Avril et al., 2016). Dietary cholesterol therefore appears to increase the risk of developing active PTB when taken in higher amounts. Our way of assessing dietary intake like all studies using FFQ was subject to recall bias. These findings are therefore to be confirmed by further studies.

Levels of TC, HDL-C and estimated cholesterol intakes differed between categories of smear grades. Levels of TC, HDL-C and LDL-C correlated negatively with degree of smear grading. Similar correlations had also been reported inother studies. A strong correlation was reported for HDL-C (Akpovi et al., 2013; Dharamveer 2015) and TC (Deniz et al., 2007; Dharamveer 2015). TG despite showing no significant correlation with smear grading in this studywas found to have a negative correlation in other study (Dharamveer 2015). Serum lipids were shown to get lower with severity of PTB (Rao 2009). Similar correlations were also found between CT scan severity of the disease and smear grading (Mohamed 2014). It's therefore likely that the correlation between serum lipids and smear grading are both indicating disease severity. It should also be noted that the degree of the oxidative stress and the levels of proinflammatory cytokines may also differ with the extent of the smear grading creating the apparent correlation.

Dietary cholesterol intake was shown in this study to positively correlate with smear grading (Table 7) indicating that higher intake of cholesterol before the development of disease was associated with higher smear grade at diagnosis. This finding may look to contradict the findings of one study that had reported cholesterol rich diet accelerates the rate of sputum sterilization on ATT (PerezGuzman et al., 2005). But the association of our study is limited to the state before starting ATT and thereby not accounting for the possible synergic effect that dietary cholesterol may have some with ATT (Iyamu 
et al., 2014). In support of our findings, there are reports on animal studies that have shown high cholesterol diet supplementation to mice caused a significant increase in the bacterial load of the host (Schäfer et al., 2009; Martens et al., 2008). If degree of smear positivity could be seen as an indicator of the bacterial load the finding of our study provide an epidemiological support for such relations.

The progress of disease from the chronic latent stage to active disease has been repeatedly shown to occur with the accumulation of foamy macrophages formed by the intracellular accumulation of LDL-C (Peyron et al., 2008). Detailed molecular and immunohistochemical analysis of human TB patients biopsy samples showed that the progress of disease was associated with increased host lipid metabolism (Kim et al., 2010). It's therefore possible that dietary cholesterol may fuel such pathologic progress of the disease. Macrophages upon infection with virulent $M t b$ had been frequently observed to accumulate lipid bodies to become foamy macrophages (FM) and are essential for allowing the pathogen to establish chronic infection (Peyron et al., 2008).The differentiation of alveolar macrophages to FM was also shown to be triggered in vitro by infection with Mtb or even by treatment with Mtb specific envelops compounds like oxygenated form of mycolic acid (Korf et al., 2005) and TDM (trehalose dimycolates) (Bowdish et al., 2009; Kim et al., 2010).Increased intakes of cholesterol from the diet may accelerate this formation of FM by increasing host cholesterol levels as dietary cholesterol was reported to increase serum total cholesterol levels (Clarke et al., 1997,Weggerman et al., 2001), but other studies restrict such effects to some groups labeled as hyperresponders showing a significant increase in LDL levels with increased intakes of dietary cholesterol (Herron et al., 2006: Chakrabarty et al.,
2004). One effect of dietary cholesterol after being absorbed is that higher amounts of cholesterol being transported in chylomicron remanants could increase the cholesterol pool of the liver after it is taken up by LDL receptors (Van der et al., 2013). This could intern down regulate the expression of LDL receptors resulting in decreased plasma LDL uptake and thereby increase plasma LDL levels (Berg et al., 2000). The increase of the available plasma LDL levels could then play the role of accelerating the formation of FM as Mtb can cause disturbances in the lipid hemostasis of the infected macrophage (Bowdish et al., 2009; Kim et al., 2010). This could lead to increased accumulation of FM in the granulomatous lesion. FMs are now thought to be key players in sustaining persistent infection and as primary drivers of pathology leading to cavitation and transmission (Russell et al., 2009). FMs were observed in necrotic lesions and their presence correlated with the onset of necrosis (Hunter et al., 2007; Welsh et al., 2010).

The main strengthens of this study include that it was done in humans and included the analysis of both serum and dietary cholesterol. The comparison was also done with subjects having a comparable nutritional statues also matched for age and sex. This study also included the comparison of newly diagnosed cases with those on treatment together with the assessment of the correlation with the extent of smear positivity. The limitations of this study include the use of smear grading of sputum samples of participants to estimate the levels of bacterial load in the specimens. More sensitive and specific culturing and molecular methods were not used. The study also used selfreported dietary intakes which may be subject to both recall and measurement errors. The presence of latent infection in the controls was also not investigated by specific tests. The study was also done in a short time 
interval which has limited its sample size to give more generalizable results. The estimates of cholesterol intake were not adjusted for energy intake as recommended by Willett (1997) because assessment of calorie intake was beyond the scope of our food frequency questionnaire which was specifically designed to assess cholesterol intake. Although the frequency of consumption for several food groups was assessed and used to adjust for the intake of other nutrients exact intake of other nutrients was also not assessed. The authors recommend further prospective cohort studies that evaluate the association of baseline serum cholesterol levels and dietary intake of cholesterol with the development of active PTB to specifically address the issue.

The present study appears to support the current trend in research showing the negative effects of dietary cholesterol due the intimate relations of cholesterol with the pathogen. This study has shown that the known adverse effects of dietary cholesterol rather than the assumed positive effects may be operating in the actual human population.

Serum cholesterol on the other hand was found to be low having a negative correlation with the extent of bacterial load of the sputum sample in the newly diagnosed cases. It may therefore be possible that increased levels of dietary cholesterol could increase the risk of developing active tuberculosis. Serum levels on the other hand may only have diagnostic significance as they appeared to alter with disease severity.

\section{Acknowledgment}

The authors are grateful to Mr. Oliver for his assistance in the statistical analysis of the data and Mr. Tsegezeab Goje and Eyob for helping in getting the ethical clearance for the work. The authors are also thankful to the lab technicians in clinical laboratory department at National Health Laboratory and in bacteriology and phlebotomy departments at Halibet and Orrota hospitals.

\section{References}

Agokpovi D. C, Gbaguidi L.H. Senami, Anago E., Affolabi D., Dougnon T.V., Faihun F. and Anagonou S.2013. Tuberculosis treatment raises total cholesterol level and restores high density lipoprotein cholesterol (HDLC) in patients with pulmonary tuberculosis. Afr. J. of Biotechnol; 12 (41) : 6019-6024.

Akiibinu O Moses, Ogunyemi O Emmanuel, Arinola O Ganiyu, Adenaike A Fidelis, Adegoke O Dickson. 2008. Assessment of antioxidants and nutritional status of pulmonary tuberculosis patients in Nigeria. Eur J Gen Med; 5(4): 208-211.

Avril Z Soh, Cynthia BE Chee, Yee-Tang Wang, Jian-Min Yuan, and WoonPuayKoh, 2016. Dietary Cholesterol Increases the Risk whereas PUFAs Reduce the Risk of Active Tuberculosis in Singapore Chinese; J Nutr: 146:10931100.

Bendel RB and Afifi AA.1997 Comparison of stopping rules in forward regression. Journal of the American Statistical Association., 72: 46-53.

Berge, K.E., Tian, H., Graf, G.A., Yu, L., Grishin, N.V., Schultz, J., Kwiterovich, P., Shan, B., Barnes, R. and Hobbs, H.H. 2000. Accumulation of dietary cholesterol in sitosterolemia caused by mutations in adjacent ABC transporters. Science, 290, 1771-1775.

Bowdish DM,SakamotoK, and Kim MJ. 2009. MARCO, TLR2, and CD14 are required for macrophage cytokine responses to mycobacterial trehalose dimycolate and Mycobacterium tuberculosis. PLoSPathog. 5: e1000474

Carlos Scherr and Jorge Pinto. 2010. Ribeiro 
Fat Content of Dairy Products, Eggs, Margarines and Oils: Implications for Atherosclerosis Arq Bras Cardiol. [online] ahead print, PP.0-0

Chakrabarty G, Bijlani R, Mahapatra S, Mehta N, Lakshmy R, Vashisht S, andManchanda S. 2004. The effect of ingestion of egg on serum lipid profile in healthy young free-living subjects. Indian $\mathrm{J}$ of Physiol and Pharmacol; 46:492-498.

Chastellier, C., and L. Thilo. 2006. Cholesterol depletion in Mycobacterium avium-infected macrophages overcomes the block in phagosome maturation and leads to the reversible sequestration of viable mycobacteria in phagolysosomederived autophagic vacuoles. Cell Microbiol. 8:242-256.

Clarke R, Frost C, Collins R, Appleby P, and Peto R. 1997 Dietary lipids and blood cholesterol: quantitative meta-analysis of metabolic ward studies. British Medical Journal; 314 (7074): 112-117.

Dharamveer Yadav. (2015). Study of lipid profile in pulmonary tuberculosis patients and relapse cases in relation with disease severity -A pilot study. International J Sci. Appl.Resear. 2(1): 41-50.

Dutta NK, Bruiners N, Pinn ML, Zimmerman MD, Prideaux B, Dartois V, Gennaro ML, Karakousis PC. 2016. Statin adjunctive therapy shortens the duration of TB treatment in mice J. Antimicrob Chemother.pii: dkw014.

Dye C and Williams BG. 2010. The population dynamics and control of tuberculosis. Science; 328: 856-861.

FigenDeveci, H. Handan Akbulut, Teyfik Turgut, and M. HamdiMuz. 2005. Changes in Serum Cytokine Levels in Active Tuberculosis with Treatment: Mediators Inflamm. 5: 256-262.

Heinrich PC, Castell JV and Andus T.1990.Interleukin-6 and the acute phase response. J.Biochem., 265:621636.

Herron K, McGrane M, Waters D, Lofgren I, Clark R, Ordovas J and Fernandez M. 2006. The ABCG5 polymorphism contributes to individual responses to dietary cholesterol and carotenoids in eggs. J. Nutr. 136:1161-1165.

Hosmer DW and Lemeshow S.1999. Applied Survival Analysis: Regression Modeling of Time to Event Data., New York: $2^{\text {nd }}$ edn. JohnWiley\&Sons.Inc. pp-16-66.

Hosmer DW and Lemeshow S.2000. Applied Logistic Regression., New York:3rdedn. JohnWiley\&Sons.Inc. pp-227-242.

Hunter RL, Jagannath C and Actor JK. 2007. Pathology of postprimary tuberculosis in humans and mice: contradiction of long-held beliefs. Tuberculosis (Edinb). 87: 267-78.

Iribarren C, Jacobs DR and Sidney S. 1998. Cohort study of serum total cholesterol and in- hospital incidence of infectious diseases. Epidemiol Infect. 121:335-47.

Iyamu O.A., Ugheoke J.E, Ozor M.O, Airhomwanbor K.O, Eidangbe A.P., Idehen I.C, Okhiai O andAkpede N. 2014. The Effect of Nutritional Lipid Supplementation on Serum Lipid Levels and Effectiveness of Antitubercular Chemotherapy American Journal of Biomedical Research. 2: 36-41.

Kassim M. Sultan, Muhammed. W. Alobaidy, Adnan M. AL-Jubouri, Azher Abbas Naser and Hamza. A. AL-Sabah. 2012. Assessment of Body Mass Index and Nutritional Status in Pulmonary Tuberculosis Patients. J Fac Med Baghdad. 54: 204-208

Kim MJ, Wainwright HC, Locketz M, Bekker LG and Walther GB, 2010. Caseation of human tuberculosis granulomas correlates with elevated host lipid metabolism. EMBO Molecular Medicine 2(7): 258-74. 
Korf, J., Stoltz, A., Verschoor, J., De Baetselier, P., and Grooten, J. 2005. The Mycobacterium tuberculosis cell wall component mycolic acid elicits pathogen-associated host innate immune responses. Eur. J. Immunol. 35:890-900.

Martens GW, Arikan MC, Lee J, Ren F, Vallerskog $\mathrm{T}$ and Kornfeld H.2008. Hypercholesterolemia impairs immunity to tuberculosis. Infect Immun; 76: 3464-72.

Mickey J and Greenland S. 1989. A study of the impact of confounder-selection criteria on effect estimation. American Journal of Epidemiology., 129: 125137.

Mohammad Reza Bolursaz, PayamMehrian, E Farahnaz Aghahosseini, Ferial Lotfian, SoheilaKhalilzadeh, Nooshin Baghaie, Maryam Hassanzad, and Ali Akbar Velayati.2014. Evaluation of the relationship between smear positivity and high-resolution $\mathrm{CT}$ findings in children with pulmonary tuberculosis: Pol J Radiol; 79: 120-125.

Pérez-Guzmán C, Vargas MH Torres-Cruz A and Villarreal-Velard H.2002. Hypocholestrelemia a frequent finding associated to tuberculosis, Rev Inst Nac EnfRepar. 15(1): 7-11.

Perez-Guzman C, Vargas MH, Quinonez F, Bazavilvazo N and Aguilar A. 2005. A cholesterol-rich diet accelerates bacteriologic sterilization in pulmonary tuberculosis. Chest, 127: 643-651.

Pérez-Guzmán C, Vargas MH, Salas-Mártir C, Trejo-Santacruz T, Gallegos-Discua C and Flores-López F. 2008. Lipid profile in household contacts of patients with pulmonary tuberculosis. Rev Med Inst Mex Seguro Soc., 46: 247-252.

Pérez-Guzmán C,and Vargas M.H.2006. Hypocholesterolemia: a major risk factor for developing pulmonary TB. Med Hypotheses, 66(6): 1227-1230.
Peyron P., J. Vaubourgeix, Y. Poquet, F. Levillain, C. Botanch, F. Bardou,M. Daffe, J.F. Emile, B. Marchou, P.J. Cardona, C. de Chastellier and Altare. F. 2008. Foamy macrophages from tuberculous patients' granulomas constitute a nutrient-rich reservoir for M. tuberculosis persistence. PLoSPathog. 4(11): e1000204.

RaoSukhesh.2009. Serum Cholesterol, HDL, LDL Levels in Pulmonary Tuberculosis: A Clinico-Radiological Correlation and Implications: Infectious Diseases in Clinical Practice:17: 99-101.

Russell DG, Cardona PJ, Kim MJ, Allain S, and Altare F.2009.Foamy macrophages and the progression of the human tuberculosis granuloma. Nat Immunol. 10: 943-948.

Samuel O. O, Adeyemi A. A, OlusegunT.O, NihinladeR.O.andAyokunleE.2013. Oxidative Stress and Lipid Profile Status in Pulmonary Tuberculosis Patients in South Western Nigeria: Greener J of Medical Sciences, 3(6): 228-232.

Sasaki Y., Yamagishi F., andYagi T., 1999. A case of pulmonary tuberculosis with pancytopenia accompanied to bone marrow gelations transformation. Kekkaku, 74: 361-364.

Schäfer G, Guler R, Murray G, Brombacher F, and Brown GD.2009. The Role of Scavenger Receptor B1 in Infection with Mycobacterium tuberculosis in a Murine Model. PLoSOne, 24: 4(12) e8448.

Scherr C, and Ribeiro JP.2009 Cholesterol and fats in Brazilian foods: implications for prevention of atherosclerosis. Arq Bras Cardiol., 92(3): 180-5.

Simons K, and Toomre D.2000. Lipid rafts and signal transduction. Nat Rev Mol Cell Biol., 1: 31-39.

Tesfaye Madebo, BerntLindtjørn, PålAukrust, and Rolf K Berge.2003.Circulating 
antioxidants and lipid peroxidation products in untreated tuberculosis patients in Ethiopia: Am J Clin Nutr.78:117-22.

Van der Wulp MY, Verkade HJ, andGroen AK. 2013 Regulation of cholesterol homeostasis.Mol Cell Endocrinol.368:1-16.

Van Gameren MM, Willemse PH and Mulder NH.1994. Effects of recombinant human interleukin-6 in cancer patients: a phase I-II study. Blood; 84:1434-41.

Weggemans R, Zock P,and Katan M.2001. Dietary cholesterol from eggs increases the ratio of total cholesterol to highdensity lipoprotein cholesterol in humans: a meta-analysis.Am J Clin Nutr.73:885-891.

Welsh, K.J., Risin, S.A., Actor, J.K. and Hunter, R.L.2010. Immunopathology of postprimary tuberculosis: Increased $\mathrm{T}$ regulatory cells and DEC-205-positive foamy macrophages in cavitary lesions. J. Immunol. Res. 307631 (2011).

WHO, Global tuberculosis control. Geneva: World Health Organization. 2010. http://www.whqlibdoc.who.int/publicati ons

Willett W.C, Howe G.R and Kushi L.H. 1997. Adjustment for total energy intake in epidemiologic studies: Am J Clin Nutr.65:1220S-1228S.

\section{How to cite this article:}

John Prabakaran, J., Biniam Efrem, Matiwos Araya, Mussie Mebrahtu, Henok Zeraburuk, Michael Yemane, Adiam Semere and Awet Ghebreyesus. 2017. Association of Dietary and Serum Cholesterol with Active Pulmonary Tuberculosis: A Hospital Based Study. Int.J.Curr.Microbiol.App.Sci. 6(12): 4056-4073. doi: https://doi.org/10.20546/ijcmas.2017.612.467 